\title{
Synchrotron-radiation-based perturbed angular correlations used in the investigation of rotational dynamics in soft matter
}

\author{
I. Sergueev, ${ }^{1,2, *}$ U. van Bürck, ${ }^{1}$ A. I. Chumakov, ${ }^{2,3}$ T. Asthalter, ${ }^{4}$ G. V. Smirnov,${ }^{3}$ H. Franz,${ }^{5}$ R. Rüffer, ${ }^{2}$ and W. Petry ${ }^{1}$ \\ ${ }^{1}$ Physik-Department E13, Technische Universität München, D-85748 Garching, Germany \\ ${ }^{2}$ European Synchrotron Radiation Facility (ESRF), P.O. Box 220, F-38043 Grenoble, France \\ ${ }^{3}$ Russian Research Center "Kurchatov Institute," 123182 Moscow, Russia \\ ${ }^{4}$ Physikalische Chemie II, Universität Stuttgart, D-70569 Stuttgart, Germany \\ ${ }^{5}$ Hamburger Synchrotronstrahlungslabor (HASYLAB), DESY, D-22603 Hamburg, Germany
}

(Received 20 June 2005; revised manuscript received 10 November 2005; published 12 January 2006)

\begin{abstract}
A new method, synchrotron-radiation-based perturbed angular correlations, was applied to study rotational dynamics of Mössbauer atoms in soft condensed matter, using incoherent nuclear resonant scattering of synchrotron radiation. A theory was developed that describes the correlations for the scattering by an ensemble of randomly oriented spins under the influence of rotational relaxation. In a feasibility study a molecular glass former doped by probe molecules was investigated above the glass transition up to the normal liquid state. A comparison of the obtained rotational relaxation rates with data from dielectric spectroscopy shows that the probe molecules reproduce the dynamics of the glass former.
\end{abstract}

DOI: 10.1103/PhysRevB.73.024203

PACS number(s): 61.43.Fs, 23.20.En, 76.80.+y, 64.70.Pf

\section{INTRODUCTION}

Mössbauer spectroscopy (MS) is a high-resolution spectroscopic method widespread in solid state physics and many other fields of science. ${ }^{1,2}$ It relies on recoilless emission and absorption of nuclear resonant $\gamma$ radiation, the probability of which is given by the Lamb-Mössbauer factor $f_{L M}$. This factor vanishes for high transition energies or soft surroundings of the resonant nuclei, thus limiting the applicability of MS. This limit is a painful hindrance for MS studies of hyperfine interactions and dynamics in soft matter like liquids, biological compounds, polymers, and plastic or liquid crystals (for reviews and original work see, e.g., Refs. 3-8 and references therein).

The natural extension of MS to the limit of vanishing $f_{L M}\left(f_{L M} \sim 0\right)$ would be the method of time differential perturbed angular correlations (TDPAC), ${ }^{9,10}$ which does not depend on the state of binding of the atom. However, more complicated demands, in particular the necessity of a suitable cascade, ${ }^{11}$ make TDPAC on most Mössbauer atoms ineffective.

The spatially incoherent channel of nuclear resonant scattering offers a way out of these difficulties via synchrotronradiation- (SR) based perturbed angular correlations (SRPAC). ${ }^{12,13}$ SRPAC can be considered as a scattering variant of TDPAC. In TDPAC the intermediate nuclear level is populated from above via a cascade of preceding nuclear transitions after decay of a radioactive parent. In SRPAC, on the contrary, the level is excited from below from the ground state during incoherent, single-nucleus resonant scattering of SR (see Fig. 1). ${ }^{14-16}$ This scattering channel avoids, in contrast to TDPAC, any chemical or electronic aftereffects. Directional selection and timing by the first detector in TDPAC are replaced in SRPAC by the direction and the timing of the incident SR flash. In both methods the interference of indistinguishable paths via an intermediate nuclear level split by magnetic dipole and/or electric quadrupole interaction allows one to investigate hyperfine interactions and spin dynamics. By contrast to MS and TDPAC, in SRPAC there is no need for radioactive parents. Hence, SRPAC can be applied to all nuclei with isomeric state with energies attainable by SR, i.e., so far for practical reasons with energies up to $150 \mathrm{keV}$. For the sake of brevity we shall call all these nuclei Mössbauer nuclei. In addition to this wider applicability of SRPAC, also the contrast of the SRPAC signals might be much larger than for TDPAC since unfavorable transitions to the excited states from above can be avoided. ${ }^{11}$

SRPAC has been briefly treated theoretically in the late 1970 s, ${ }^{14}$ in parallel with the first experimental demonstration

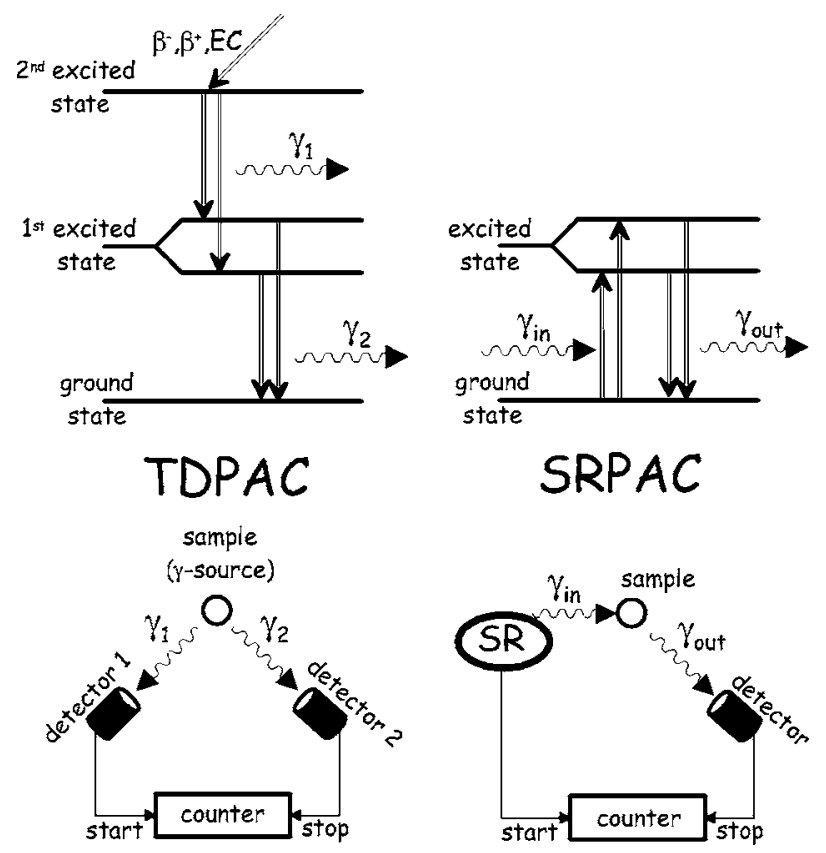

FIG. 1. Schemes of the principle and of the experimental setup for TDPAC (left side) and SRPAC (right side). 
of TDPAC-like atomic resonant scattering of SR in the VUV. ${ }^{17}$ Nuclear resonant scattering of SR (Ref. 18) was verified only years later. Here the intense channels of collective, spatially coherent scattering have been investigated first: nuclear Bragg scattering (NBS) ${ }^{19}$ and nuclear forward scattering (NFS). ${ }^{20}$ Both channels rely on recoil-free scattering and break down for $f_{L M} \sim 0$. Spatially incoherent nuclear scattering ${ }^{21}$ became accessible for investigation of the solid state with the advent of the powerful third generation SR storage rings ESRF, APS, and SPring-8. Besides timeintegral applications, ${ }^{22-24}$ first time-differential studies have been performed, where typical magnetic quantum beats have been demonstrated in single-nucleus scattering by pure iron. ${ }^{25}$ Recently, spatially incoherent scattering was applied to study site-specific phonon densities of states. ${ }^{26}$

In the present study we have applied SRPAC for the first time to investigate hyperfine interactions and dynamics of Mössbauer atoms in the case of $f_{L M} \sim 0$. Preliminary results of our work have been presented elsewhere. ${ }^{12,13,27}$ The paper is organized as follows: In Sec. II we derive an expression to describe the intensity of SRPAC based on the general theory of angular correlations. In Sec. III we describe the model of rotational relaxation and derive an analytical expression that describes its influence on SRPAC. The obtained theory is applied to analyze our experimental data on the molecular glass former dibutyl phthalate doped with $5 \%$ of ferrocene enriched in ${ }^{57} \mathrm{Fe}$. The experimental aspects of the method and the data evaluation are discussed in Sec. IV. The obtained rotational relaxation rates of the probe molecules are discussed in Sec. V and are compared with the data from dielectric spectroscopy on pure dibutyl phthalate.

\section{GENERAL FORMALISM}

Nuclear resonant scattering of pulsed SR can be treated as a sequence of three stages: excitation of the ensemble of resonant nuclei via absorption of the incoming radiation, time evolution of the ensemble in the excited state, and subsequent radiative decay of this state. Depending on the type of the decay, the scattering can be spatially coherent or incoherent. If the nuclear system returns to its original state, the scattering is spatially coherent, because no mark is left telling on which nucleus the scattering actually occurred. Here all the nuclei in the sample can be excited with equal probability so that the nuclear excitation is distributed over the whole nuclear ensemble where all scattering contributions have to be summed up on the amplitude level. Incoherent scattering takes place when the state of a particular nucleus (atom) after the scattering process is different from the original state. Then the nucleus on which the scattering occurred can be identified, at least in principle, since it was tagged by that change of state. For the decay with reemission of resonant radiation the original and final nuclear states can differ, e.g., by the spin state. Hence spin-flip scattering is incoherent. But also the phonon-assisted inelastic scattering is spatially incoherent, even though the phonon is a collective excitation. Nuclear and phonon lifetimes, however, differ by orders of magnitude, and therefore the coherence in the relevant time window is destroyed. Another possibility is the decay with internal conversion when the resonant energy is transferred to the electronic shell with subsequent emission of atomic fluorescence. This atomic fluorescence, however, does not contain information about the nuclear intermediate state. Therefore, in SRPAC we record only the nuclear resonance fluorescence.

The theoretical description of the time and angular dependence of the intensity of this incoherent scattering channel (henceforth called SRPAC signal) can be derived from the scattering amplitude ${ }^{14}$ similar to the derivation of the coherent scattering intensity from the coherent scattering amplitude. ${ }^{14,28}$ Another approach, used, e.g., in TDPAC, is based on the concept of perturbation factors describing the time evolution of the intermediate excited nuclear state. Here the scattered intensity is derived directly. Both methods lead to the same result, but the description of relaxation phenomena is more easily incorporated into the second approach. For this reason it will be adapted here to describe SRPAC. For simplification we will consider only the case where the nuclear spins are randomly oriented in the sample which is the usual case in soft condensed matter. One should keep in mind, however, that according to the polarization dependence of nuclear interaction, only a subensemble of nuclei with preferable direction of the nuclear spin is observed in TDPAC and SRPAC. The temporal and angular correlation between two emitted photons in TDPAC (more precisely, directional TDPAC) according to Frauenfelder, Steffen, and Alder $^{29-31}$ is given by an expansion

$$
W(\phi ; t) \propto \mathrm{e}^{-t / \tau_{0}}\left[1+A_{22} G_{22}(t) P_{2}(\cos \phi)+\cdots\right],
$$

where $\phi$ is the angle between two emitted $\gamma$-photons; the anisotropy coefficient $A_{22} \equiv A_{2}(1) A_{2}(2)$ is defined by the first and second nuclear transitions (see Fig. 1) and depends on the values of the nuclear spins and the multipolarity of the transitions; $P_{2}(\cos \phi)$ is the Legendre polynomial; and $\tau_{0}$ is the life time of the excited state. The perturbation factor $G_{22}(t)$ describes the time evolution of the excited nuclear state. Its shape depends on the hyperfine interactions and is given in the static case by

$$
G_{k k}^{(0)}(t)=\sum_{m, m^{\prime}}\left(\begin{array}{ccc}
I & I & k \\
m^{\prime} & -m & m-m^{\prime}
\end{array}\right)^{2} \exp \left[l\left(E_{m^{\prime}}-E_{m}\right) t / \hbar\right],
$$

where $k$ is the running index of expansion of Eq. (1), $m, m^{\prime}$ denote sublevels of the excited state with spin $I$ and corresponding energies $E_{m}, E_{m^{\prime}}$, and large brackets denote Wigner $3 \mathrm{jm}$ symbols, the definition of which can be found, e.g., in Ref. 32.

The emission of the first $\gamma$ photon in TDPAC is replaced in SRPAC by the absorption of the incoming SR photon. This, however, does not change the shape of Eq. (1). A more important distinction is that in SRPAC the SR photon is linearly polarized in the plane of the synchrotron ring whereas in directional TDPAC the photons are assumed to be unpolarized. This, in general, results in a strong modification of Eq. (1). However, a simple and elegant solution exists in the case when the nuclear transition from the ground state to the excited state is a pure magnetic dipole (M1) transition. This 


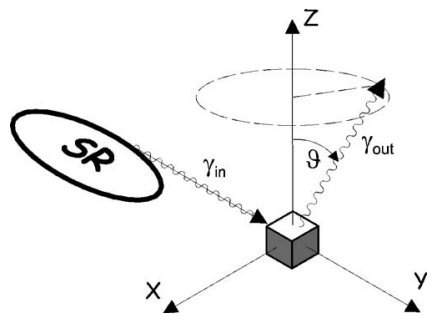

FIG. 2. Geometry of SRPAC. The $x$ and $y$ axes lie in the plane of the storage ring.

is approximately the case for a large number of Mössbauer isotopes. By this condition the expansion in Eq. (1) is reduced to two terms. In the Appendix we show that the SRPAC signal for randomly oriented nuclear spins is then given as [Eq. (A12)]

$$
I(\vartheta ; t)=I_{0} \mathrm{e}^{-t / \tau_{0}}\left[1-2 A_{22} G_{22}(t) P_{2}(\cos \vartheta)\right],
$$

where $\vartheta$ is the angle between the scattered photon and the magnetic polarization of the incoming photon (see Fig. 2) and $A_{22} \equiv A_{2}(1) A_{2}(1)$ is the anisotropy coefficient for SRPAC, where nuclear excitation and deexcitation are described by the same parameter. The shape of Eq. (3) is similar to Eq. (1). The time dependence in both cases is described by the same perturbation factor $G_{22}(t)$ which, however, is twice as pronounced for SRPAC as for TDPAC due to the factor of -2 in front of the anisotropy coefficient. This originates from the linear polarization of SR. On the other hand, the TDPAC correlation function depends on the angle between the directions of emission of the two correlated photons of the cascade, whereas the SRPAC intensity depends on the angle between the direction of the magnetic polarization of the incoming linearly polarized photon and the direction of reemission of the scattered photon. The magnetic polarization of the SR photon is directed perpendicular to the $\mathrm{SR}$ ring, i.e., parallel to the $z$ axis as shown in Fig. 2. This leads to isotropic scattering around the vertical axis for SRPAC, whereas for TDPAC the scattering is isotropic around the emission direction of the first $\gamma$ photon.

The nontrivial part in Eq. (3) factorizes into an anisotropy coefficient $A_{22}$, a geometrical factor $P_{2}(\cos \vartheta)$, and a perturbation factor $G_{22}(t)$. The anisotropy coefficient $A_{22}$ is given in Table I for several Mössbauer isotopes used for nuclear resonant scattering.

The angular dependence of the SRPAC intensity is defined by the Legendre polynomial $P_{2}(\cos \vartheta)$. It has its maxi-

TABLE I. The anisotropy coefficient $A_{22}$ for several Mössbauer isotopes used for nuclear resonant scattering. $I_{g}, I_{e}$ are the spins of the ground and excited states.

\begin{tabular}{cccc}
\hline \hline Isotope & $I_{g}$ & $I_{e}$ & $A_{22}$ \\
\hline${ }^{57} \mathrm{Fe},{ }^{119} \mathrm{Sn}$ & $1 / 2$ & $3 / 2$ & $1 / 4=0.25$ \\
${ }^{61} \mathrm{Ni}$ & $3 / 2$ & $5 / 2$ & $7 / 50=0.14$ \\
${ }^{121} \mathrm{Sb},{ }^{151} \mathrm{Eu}$ & $5 / 2$ & $7 / 2$ & $3 / 28 \simeq 0.11$ \\
${ }^{149} \mathrm{Sm}$ & $7 / 2$ & $5 / 2$ & $1 / 56 \simeq 0.018$ \\
\hline \hline
\end{tabular}

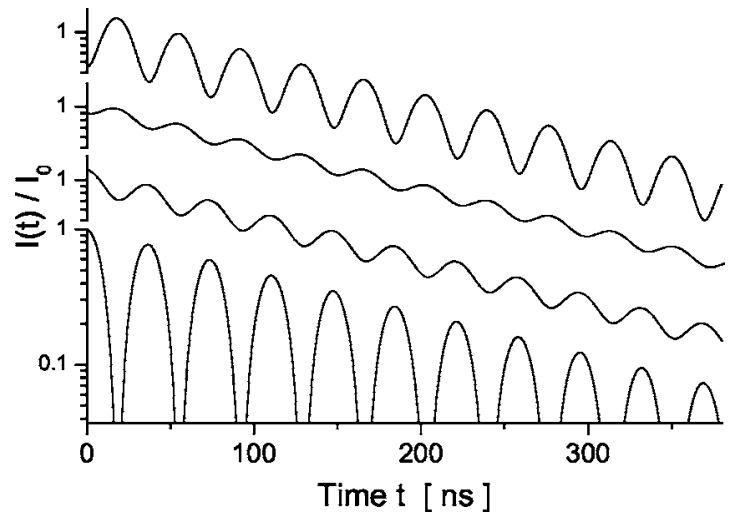

FIG. 3. Time dependence of the SRPAC signal $I(\vartheta ; t)$ (in $\log$ scale) for the angle $\vartheta$ equal to (from top) $0^{\circ}, 45^{\circ}$, and $90^{\circ}$. The bottom curve shows the time dependence of the NFS intensity. The parameters of the simulation are $\tau_{0}=141.1 \mathrm{~ns}$ and $\Omega=24 / \tau_{0}$.

mum value, 1 , along the vertical axis ( $z$ axis in Fig. 2). An increase of the scattering angle $\vartheta$ leads to a decrease of $P_{2}(\cos \vartheta)$, which becomes zero at $\vartheta \simeq 54.7^{\circ}$. At this angle the time evolution of the SRPAC intensity follows a natural decay independent of the hyperfine interactions. Further increase of the scattering angle leads to negative values of $P_{2}(\cos \vartheta)$, which becomes $-\frac{1}{2}$ in the horizontal plane.

The static perturbation factor $G_{22}^{(0)}(t)$ is defined in SRPAC by the same Eq. (2) as in TDPAC. If there is no splitting of the excited state, $G_{22}^{(0)}(t) \equiv 1$ and the SRPAC intensity follows the natural decay. In this work we will consider the perturbation of $G_{22}^{(0)}(t)$ by an axially symmetric quadrupole interaction caused by the interaction of the quadrupole moment of the excited nuclear state with an electric field gradient (EFG) produced by the surroundings. The direction of the EFG is assumed to be isotropically distributed. $G_{22}^{(0)}(t)$ for various spins can be found e.g., in Ref. 9. Here we consider the ${ }^{57} \mathrm{Fe}$ isotope with spin of the excited state $I=\frac{3}{2}$ which results in a static perturbation factor given by

$$
G_{22}^{(0)}(t)=\frac{1}{5}+\frac{4}{5} \cos \Omega t,
$$

where $\hbar \Omega$ is the quadrupole splitting of the excited state. Inserting Eq. (4) into Eq. (3) one obtains that the SRPAC signal is described by a natural decay modulated by quantum beats of a single frequency

$$
I(\vartheta ; t) \propto \mathrm{e}^{-t / \tau_{0}}[1+K(\vartheta) \cdot \cos \Omega t],
$$

where $K(\vartheta)$ is the contrast of the quantum beats, which is $K \simeq-0.44$ for $\vartheta=0^{\circ}, 180^{\circ}$ and $K \simeq 0.19$ for $\vartheta=90^{\circ}$. The time dependence of the SRPAC intensity for various scattering angles is shown in Fig. 3.

Also the intensity of NFS is described in the kinematical approximation by Eq. (5) with $K=1$. The similarity of coherent (NFS) and incoherent (SRPAC) scattering in this case is due to the fact that the ground state of ${ }^{57} \mathrm{Fe}$ with $I=\frac{1}{2}$ is not split by the quadrupole interaction. The difference between SRPAC and NFS becomes more pronounced for magnetic dipole interaction. Here the time dependence of coherent and incoherent nuclear resonant scattering is quite different ${ }^{14,25}$ 
since spatially coherent scattering involves interference of scattering paths via many nuclei in different ground states, whereas incoherent scattering proceeds only via single nuclei which are each in a particular ground state.

\section{INFLUENCE OF RELAXATION}

The static perturbation factor of Eq. (4) can be modified by relaxation, which results from fluctuations of the hyperfine field. Here we will describe the effect of relaxation caused by stochastic molecular reorientations that take place in the liquid state.

We assume that the EFG on the iron atom is defined solely by the molecule where it is embedded. A rotation of the molecule leads to a rotation of the EFG which is the axis of the quadrupole interaction. Hence the time evolution of the perturbation factor is determined by two processes: a precession of the nuclear spin around the EFG with the frequency $\Omega$ and a stochastic rotation of the EFG with a characteristic jump or relaxation rate $\lambda$. The ratio of $\Omega$ and $\lambda$ defines the characteristic shape ${ }^{33}$ of $G_{22}(t)$. If the relaxation is slow, i.e., $\lambda \ll \Omega$, the time evolution of $G_{22}(t)$ is mainly described by a precession around the EFG which leads to quantum beats in the intensity. The averaging due to the slow stochastic rotation of the EFG causes a damping of the oscillations. In the opposite case, when $\lambda \gg \Omega$, the change of the EFG direction is so fast that no precession can take place. Then the ensemble of spins slowly relaxes to the isotropic state.

The approach for the calculation of the time evolution of the nuclear spin states driven by hyperfine interactions and stochastic reorientation of the quantization axis was developed by Blume ${ }^{34}$ as a generalization of the Kubo-Anderson model. ${ }^{35}$ This theory was applied for the calculation of the TDPAC perturbation factor by Dattagupta, ${ }^{36}$ Lynden-Bell, ${ }^{37}$ and Winkler. ${ }^{38}$ The formalism is based on the stochastic theory approach where one separates the full system into the probe (nucleus) and its surroundings, which can be replaced by an effective medium called the heat bath governed by stochastic motions. The heat bath and probe are connected by hyperfine interactions. The heat bath can be described by one parameter (in our case the direction of the EFG), the time evolution of which is governed by the stochastic motion. In the frame of the model, the stochastic motion is assumed to be a stationary Markov process, i.e., a process without memory.

We assume that the reorientation is isotropic and proceeds by finite angular jumps. The main parameter which defines the relaxation process is the average dwell time $\tau$ during which the molecule resides in a given orientation. The corresponding frequency parameter is the relaxation rate $\lambda$ $=1 / \tau$. The angular jump is assumed to have a random magnitude so that after the jump the molecule can find itself oriented in any direction independent of the previous state. This model is called the strong collision model (SCM) or random jump model..$^{39,40}$ The perturbation factor for TDPAC in this model was derived by Dattagupta. ${ }^{36}$ The advantage of the application of this model to SRPAC on ${ }^{57} \mathrm{Fe}$ is that for spin $I=\frac{3}{2}$ an analytical expression for $G_{22}(t)$ can be obtained.
We start our analysis from the Laplace image of the perturbation factor

$$
G_{22}(p)=\int_{0}^{\infty} \mathrm{d} t G_{22}(t) \mathrm{e}^{-p t},
$$

where $p$ is the frequency variable of the Laplace transformation. In the static case the Laplace transformation of Eq. (4) leads to

$$
G_{22}^{(0)}(p)=\frac{1}{5}\left(\frac{1}{p}+\frac{4 p}{p^{2}+\Omega^{2}}\right) .
$$

The modification of the perturbation factor due to relaxation in the frame of the SCM can be expressed as [see Eq. (4.27) in Ref. 36]

$$
G_{22}(p)=\frac{G_{22}^{(0)}(p+\lambda)}{1-\lambda G_{22}^{(0)}(p+\lambda)} .
$$

Inserting Eq. (7) into Eq. (8) we obtain

$$
G_{22}(x)=\frac{1}{\Omega} \frac{5(x+\tilde{\lambda})^{2}+1}{5 x\left[(x+\tilde{\lambda})^{2}+1\right]+4 \tilde{\lambda}},
$$

where $x=p / \Omega$ and $\tilde{\lambda}=\lambda / \Omega$. In order to make the inverse Laplace transform to the time variable one has to expand this expression in partial fractions and therefore has to find the roots of the denominator. Since the denominator is a cubic expression of $x$, an analytical solution can be obtained. This is different from the majority of the TDPAC isotopes where, due to a spin $I=\frac{5}{2}$, the denominator is an expression of the fifth power, where no analytical solution exists.

Expanding Eq. (9) and making the inverse Laplace transformation we obtain an expression for the perturbation factor in the time domain

$$
G_{22}(t)=A \mathrm{e}^{-\alpha \Omega t}+(1-A) \mathrm{e}^{-\beta \Omega t} \cos (\gamma \Omega t)+C \mathrm{e}^{-\beta \Omega t} \sin (\gamma \Omega t),
$$

where

$$
\begin{gathered}
\alpha=\frac{2}{3} \tilde{\lambda}-s, \quad \beta=\frac{2}{3} \tilde{\lambda}+\frac{1}{2} s, \quad \gamma=\frac{\sqrt{3}}{2} r, \\
A=\frac{20(3 s+\tilde{\lambda})^{2}+36}{135\left(r^{2}+3 s^{2}\right)}, \quad C=\frac{\sqrt{3}(3 s+4 \tilde{\lambda})}{9 r}-\frac{s \sqrt{3}}{r} A, \\
s=(y+w)^{1 / 3}+(y-w)^{1 / 3}, \quad r=(y+w)^{1 / 3}-(y-w)^{1 / 3}, \\
y=\tilde{\lambda}\left(5 \tilde{\lambda}^{2}-9\right) / 135, \quad w=\sqrt{\frac{5 \tilde{\lambda}^{4}-22 \tilde{\lambda}^{2}+25}{675}} .
\end{gathered}
$$

The simulation of the perturbation factor with this expression is shown in Fig. 4 for several ratios of relaxation rate $\lambda$ and quadrupole frequency $\Omega$, with the latter being kept constant.

In order to obtain a qualitative understanding of the influence of the relaxation we consider here the limiting cases of slow and fast relaxation. 


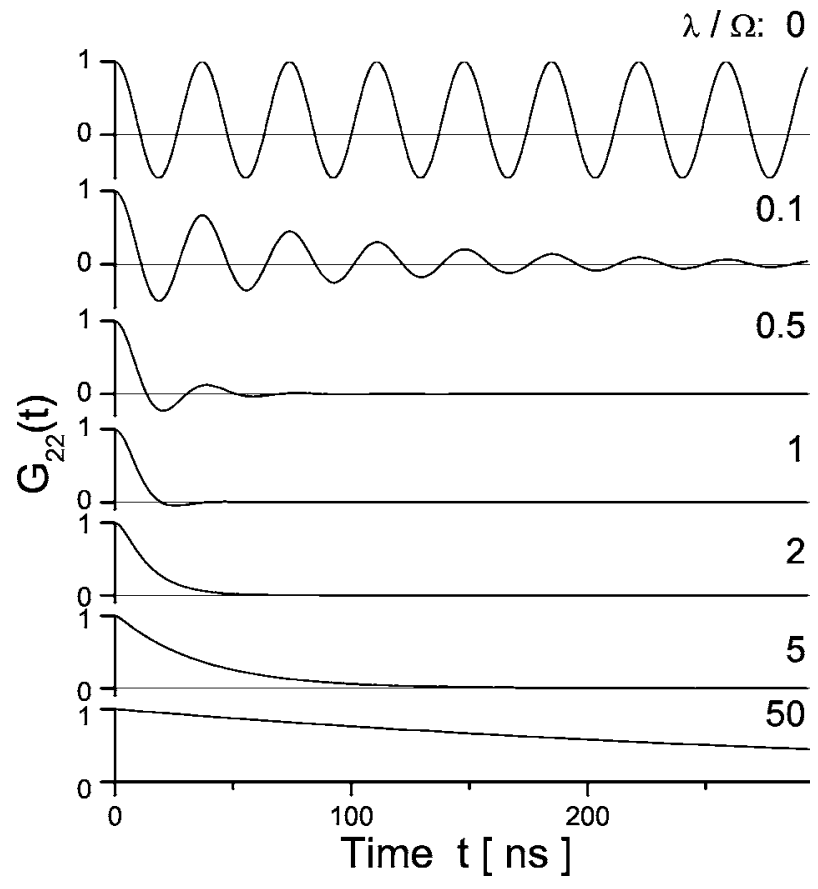

FIG. 4. Simulation of the perturbation factor $G_{22}(t)$ for various ratios of $\lambda / \Omega$ in the frame of the SCM according to Eq. (10). The parameters of the simulation are $\tau_{0}=141.1 \mathrm{~ns}$ and $\Omega=24 / \tau_{0}$.

The slow relaxation regime is defined by $\tilde{\lambda} \ll 1$ (i.e., $\lambda$ $\ll \Omega$ ). Then, up to the first order of $\tilde{\lambda}$ the perturbation factor is given as

$$
\begin{aligned}
G_{22}(t) \simeq & \frac{1}{5} \exp (-4 \lambda t / 5)+\frac{4}{5} \exp (-3 \lambda t / 5) \\
& \cdot\left(\cos \Omega_{e} t+\frac{4 \lambda}{5 \Omega} \sin \Omega_{e} t\right)
\end{aligned}
$$

with

$$
\Omega_{e}=\Omega\left(1-4 \lambda^{2} / 25 \Omega^{2}\right) .
$$

Here the second-order term in the frequency $\Omega_{e}$ is given in order to show the development of this parameter. $G_{22}(t)$ as given by Eq. (11) is similar to the static perturbation factor given in Eq. (4). The relaxation manifests itself in an exponential damping with a rate proportional to $\lambda$, in a slight change of the frequency and in the appearance of the sine term.

The physical meaning of Eq. (11) can be understood in a quasi-classical picture using the adiabatic approximation ${ }^{43}$ widely applied in TDPAC to describe the slow relaxation regime

$$
G_{22}(t) \simeq \mathrm{e}^{-\lambda t} \cdot G_{22}^{(0)}(t) .
$$

This approximation assumes that the relaxation rate is so slow that the spin precession seen from the coordinate system of the EFG is the same as in the static case. On the other hand, the direction of the EFG, as defined by the incoming photon, stochastically changes in time, which leads to the stochastic perturbation of the angular pattern of the reemis- sion. As a result, the perturbation factor is the product of the static perturbation factor $G_{22}^{(0)}(t)$ which describes a precession of the spin around the EFG and a factor $\mathrm{e}^{-\lambda t}$ which describes the correlation between the directions of the EFG at time zero and at the time of reemission, averaged over the nuclear ensemble. This correlation function is often assumed to be an exponential decay in time.

The adiabatic approximation, however, is not valid for the SCM because the instant jump of a molecule from one direction to another assumed in the SCM is not an adiabatic motion. ${ }^{41}$ At each jump of the EFG, the spin motion, which is continuous in the stationary coordinate system, experiences a jump in the coordinate system of the EFG. This leads to a perturbation of the spin precession and, due to the quantum mechanical nature of the spin variable, to a mixing of the populations of the spin states. These are so-called nonsecular effects. ${ }^{40}$ As a result, by contrast to Eq. (13), the rates of the exponential decay are different for the two terms in Eq. (11) (i.e., $-4 \lambda / 5$ and $-3 \lambda / 5$ ), and a frequency shift and an additional sine term appear. Similar results are obtained in Ref. 41. Note that the jump of the spin in the EFG coordinate system is related to the characteristic jump of the molecular reorientation, which is an essential parameter of the reorientational model. Therefore, the ratio of the rates of the two exponential decays in the perturbation factor given by Eq. (11) should allow one to distinguish between different models of reorientation. ${ }^{42}$

The fast relaxation regime is defined by $\tilde{\lambda} \gg 1$ (i.e., $\lambda$ $\gg \Omega$ ). Up to the first order of $1 / \tilde{\lambda}$ and for $\lambda t \gg 1$ the perturbation factor is given as

$$
G_{22}(t) \simeq \exp \left(-4 \Omega^{2} t / 5 \lambda\right) .
$$

In this regime oscillations are not observed any more. The perturbation factor exponentially decays in time with a rate inversely proportional to the relaxation rate. This regime is called the motional narrowing limit or Abragam-Pound limit. ${ }^{33}$ At time zero the nucleus finds itself in a state with a certain direction of the quantization axis. Its spin starts to precess around this axis, but, since the jump rate is very fast, it can hardly precess at all before the axis jumps to a new direction. Since the nuclear spin cannot follow such a rapid relaxation it sees an averaged EFG. With the increase of the jump rate, the averaged EFG decreases and therefore the rate of the spin motion decreases as well. As a result the loss of the anisotropy, which is produced by the spin motion, is slowing down (see Fig. 4). In the case of an infinitely large jump rate the ensemble of spins is decoupled from the environment and the perturbation factor stays at a constant value $G_{22}(t)=1$.

One can estimate the possible range for investigations of the relaxation rate $\lambda$ assuming a constant quadrupole frequency. Practically the minimal damping of the perturbation factor that can usually be observed in the experiment is limited to $0.1 / \tau_{0}$. This leads to a limitation from below to $\lambda$ $\gtrsim 0.1 / \tau_{0}$ and from above to $\Omega^{2} / \lambda \gtrsim 0.1 / \tau_{0}$. Taking as an example ${ }^{57} \mathrm{Fe}$ with $\tau_{0}=141.1 \mathrm{~ns}$ and assuming a quadrupole splitting of $\Omega=24 / \tau_{0}$ which is typical for ferrocene and representative for $\mathrm{Fe}^{2+}$ compounds in general, we obtain that the 


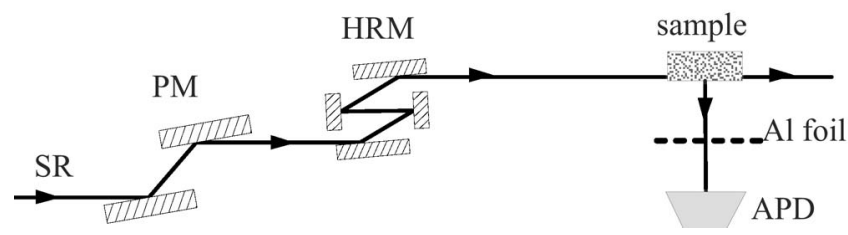

FIG. 5. Side view of the experimental setup of SRPAC with $\mathrm{PM}$ - premonochromator, HRM-high-resolution monochromator, and APD - avalanche photodiode. The axis sample-APD corresponds to the $z$ axis of Fig. 2.

relaxation rate can be studied in a range of $7 \times 10^{5}$ to $4 \times 10^{10} \mathrm{~s}^{-1}$ or, in units of relaxation time, in between $\sim 25 \mathrm{ps}$ and $1.4 \mu \mathrm{s}$. This means that a range of almost five orders of magnitude can be covered by this method.

\section{EXPERIMENTS}

As a model substance, we have chosen the molecular glass former dibutyl phthalate (DBP) doped with 5\% (mol) of ferrocene (FC) enriched to $95 \%$ in ${ }^{57} \mathrm{Fe}$. The FC molecule has an iron atom in the center of mass between two cyclopentadienyl $\left(\mathrm{C}_{5} \mathrm{H}_{5}\right)$ rings which produce an EFG and, correspondingly, a quadrupole splitting of the nuclear level at the iron site. DBP-FC has a caloric glass transition temperature $T_{g}=179 \mathrm{~K}$, the same as pure DBP. This substance has been measured by $\mathrm{MS},{ }^{44} \mathrm{NFS},{ }^{45,46}$ and nuclear inelastic scattering. ${ }^{47}$ However, due to the drastic decrease of the LambMössbauer factor $f_{L M}$, above $T_{g}$ these measurements hit an upper limit at $\sim 210 \mathrm{~K}$ for NFS and $\sim 212 \mathrm{~K}$ for MS where the countrates proportional to $f_{L M}^{2}$ and $f_{L M}$, respectively, become $\sim 0$. SRPAC, by contrast, is independent of $f_{L M}$. Therefore in this work measurements were performed up to $\sim 330 \mathrm{~K}$, i.e., far in the normal liquid state of DBP-FC.

The experiment was carried out at the nuclear resonance beamline $^{48}$ ID18 of the European Synchrotron Radiation Facility (ESRF). The filling modes of the storage ring were the 16- or 1-bunch modes with time windows of 176 and $2800 \mathrm{~ns}$, respectively. Preliminary measurements had been performed at the PETRA I beamline ${ }^{49}$ of the HASYLAB (Hamburg).

The $14.4 \mathrm{keV}$ incident radiation was monochromatized in two steps by a premonochromator and a high-resolution monochromator in order to reduce the load on the detector (see Fig. 5). While for NFS the bandwidth of 5-50 neV around the resonant energy is relevant, SRPAC can occur with absorption or emission of phonons, and a bandwidth of 100-200 meV can be used in principle. We found it convenient to employ a bandwidth of $6 \mathrm{meV}$, which covers $\sim 50 \%$ of the absorption area and which is a good compromise between the usable intensity and the load on the detector. Note that the shape of the SRPAC spectrum does not depend on the phonons that participate in the nuclear absorption process since the characteristic lifetime of the phonons is much shorter than the nuclear decay and the experimental time window.

A sample of $2 \times 4 \mathrm{~mm}^{2}$ cross section and $10 \mathrm{~mm}$ length was mounted in a closed-cycle cryostat in a copper holder

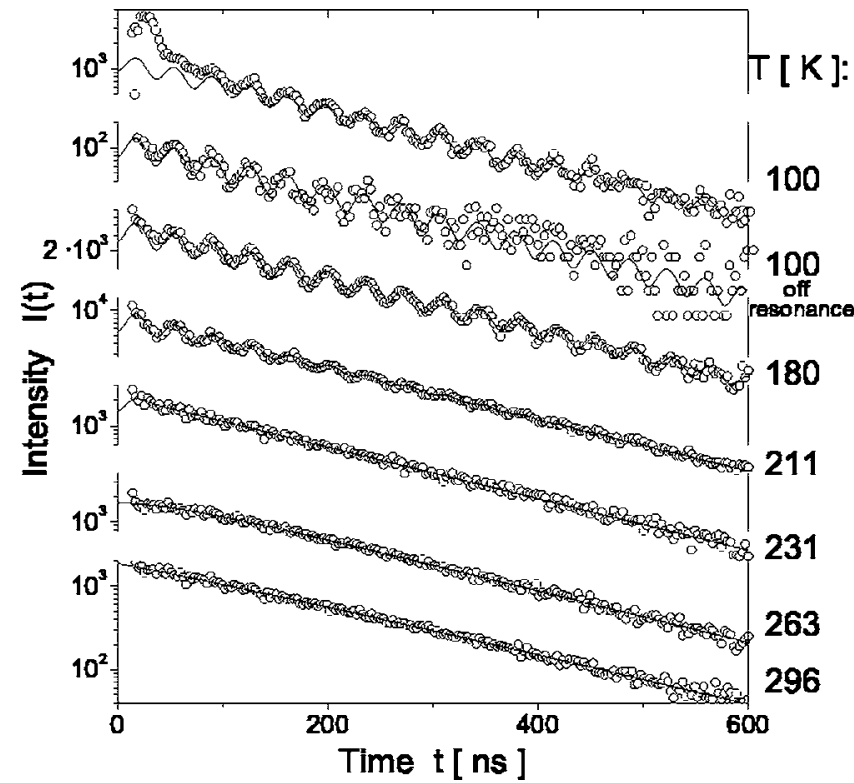

FIG. 6. Time evolution of the SRPAC signal (in log scale) for several temperatures. The solid lines are fits by the theory given by Eqs. (3) and (10).

with Kapton windows. The measurements have been performed in the temperature range 100-330 K.

The time evolution of the scattered radiation was monitored by a $10 \times 10 \mathrm{~mm}^{2}$ avalanche photodiode ${ }^{50}$ mounted in $90^{\circ}$ scattering geometry $10 \mathrm{~mm}$ below the sample. The $6.4 \mathrm{keV} K_{\alpha}$ radiation of the iron atom produced by internal conversion was eliminated by a $320 \mu \mathrm{m}$ thick Al foil (see Fig. 5). The foil reduces the $6.4 \mathrm{keV}$ radiation by a factor of $\sim 3000$, whereas the $14.4 \mathrm{keV}$ resonant radiation is reduced only by a factor of $\sim 2$. In comparison to a similar experimental setup used for the nuclear inelastic scattering technique, ${ }^{2-24}$ typical countrates in SRPAC are a factor of 50-100 lower due to angular collimation and exclusion of the dominant $6.4 \mathrm{keV}$ radiation. The observed countrate was on average $\sim 30 \mathrm{~Hz}$.

The measured time dependence of the SRPAC signal is shown in Fig. 6 for several temperatures. It follows a natural exponential decay with a lifetime $141.1 \mathrm{~ns}$ perturbed by the hyperfine interactions. At low temperatures, i.e., in the static case, the quadrupole splitting of the excited state results in pronounced quantum beats with a period of $\sim 36 \mathrm{~ns}$. These beats start with a minimum at $t=0$ and occur in antiphase to the beats observed in NFS (Ref. 46) (compare Fig. 3). The contrast of the beats is relatively high, amounting to $\sim 40 \%$.

With rising temperature, the beats are increasingly damped and finally disappear above $\sim 240 \mathrm{~K}$. At higher temperatures a smooth approach of the natural decay is observed, with the initial deviation from the natural decay being negative.

In the temperature range where the Lamb-Mössbauer factor is relatively large the SRPAC signal has a more complicated structure, as can be seen from the spectrum recorded at $100 \mathrm{~K}$ displayed in Fig. 6. At early times the signal is dominated by the additional contribution from Rayleigh scattering of the NFS intensity (NFS-Rayleigh scattering). In order to 


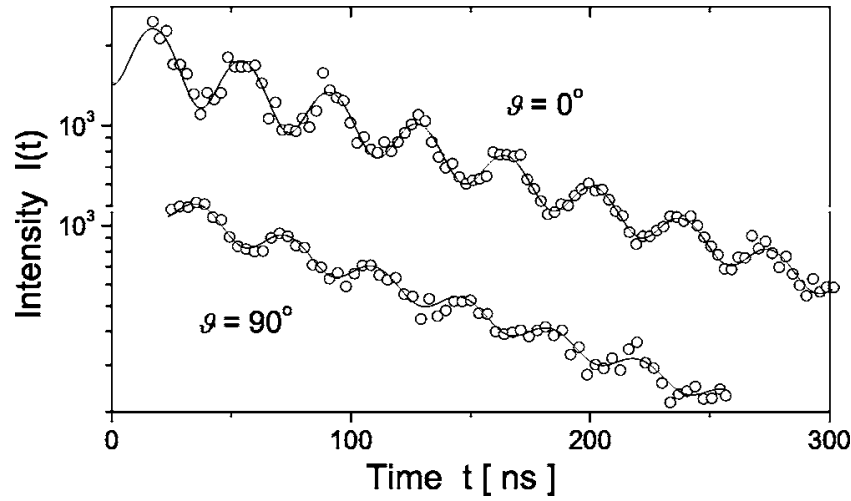

FIG. 7. Time evolution of the SRPAC signal (in log scale) measured along the $z$ axis $\left(\vartheta=0^{\circ}\right)$ and along the $x$ axis $\left(\vartheta=90^{\circ}\right)$ in Fig. 2. The solid lines are fits by the theory given by Eqs. (3) and (10).

estimate the influence of this contribution we measured the SRPAC signal at $100 \mathrm{~K}$ off resonance, as performed in Ref. 25 . Here we moved the bandwidth of the monochromator $20 \mathrm{meV}$ above the nuclear resonance, so that nuclear scattering can occur only with creation of phonons. In this case no coherent scattering exists and a pure SRPAC signal is observed. The comparison of the time evolutions measured on and off resonance (first and second curves in Fig. 6) shows that the contribution of NFS-Rayleigh scattering is important only in the first $\sim 100 \mathrm{~ns}$. This limitation of the NFSRayleigh contribution to early times is due to the speed-up of coherent scattering for large $f_{L M}$ (large effective thickness of the sample), which concentrates the coherent scattering to early times. In principle, measurements of SRPAC off resonance are useful or, at short bunch distances, even necessary to extract information about hyperfine interactions and dynamics in the case of a large $f_{L M}$. However, the countrate in such measurements is only a few percent of the countrate at resonance, which seriously limits the application of the offresonance measurements.

In order to check the angular dependence of SRPAC we have measured the scattering at $200 \mathrm{~K}$ for two directions: along the $z$ axis and along the $x$ axis in Fig. 2. The spectra are shown in Fig. 7. One can see that the quantum beats for the spectrum measured along the $x$ axis are in anti-phase to those for the spectrum measured along the $z$ axis and have roughly a twice smaller contrast, which corresponds to the theoretical prediction of Eq. (5) as illustrated in Fig. 3.

\section{RESULTS AND DISCUSSION}

The anisotropic part of the scattered intensity, also called anisotropy $2 A_{22} G_{22}(t)$, is shown in Fig. 8 for several temperatures. It was obtained from the experimental data (Fig. 6) using Eq. (3) with $P_{2}(\cos \vartheta)=1 . A_{22}$ is here the effective anisotropy coefficient, which includes the averaging of the scattering over the finite sizes of detector and sample. It is clearly seen that the time dependence of the anisotropy is different for temperatures below and above $\sim 240 \mathrm{~K}$. Below this temperature the anisotropy is described by quantum beats which are damped with a rate increasing with rising temperature. This temperature range below $\sim 240 \mathrm{~K}$ corre-

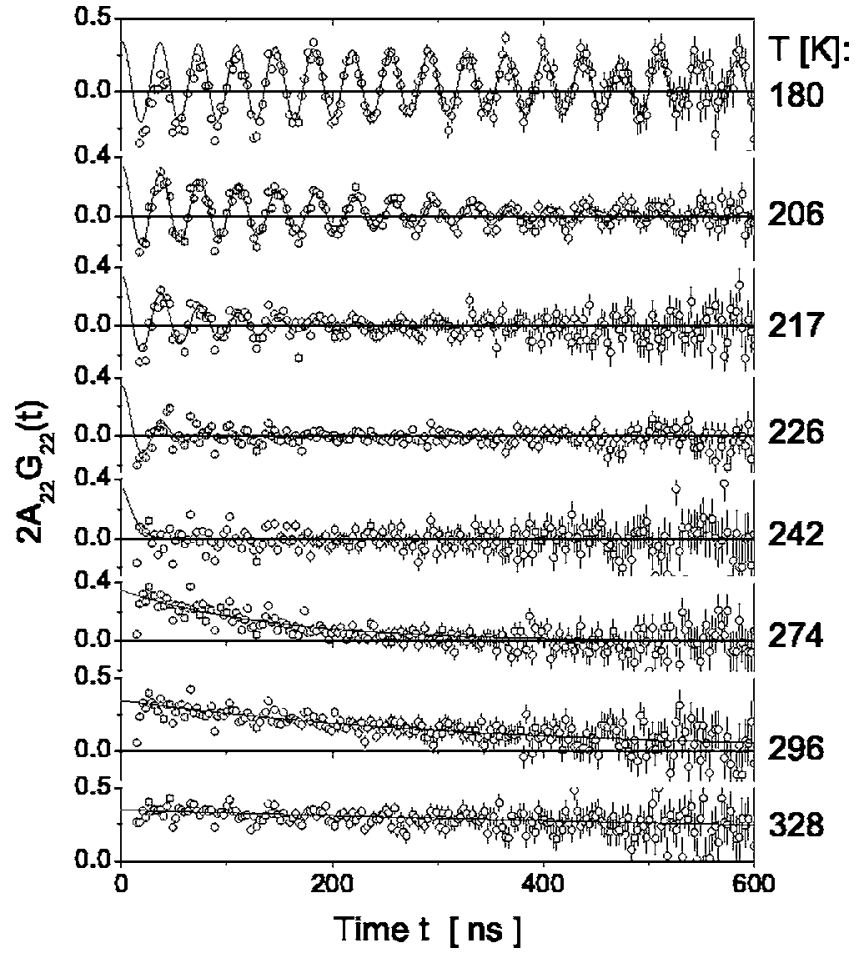

FIG. 8. Time evolution of the anisotropy $2 A_{22} G_{22}(t)$ for several temperatures. The solid lines are fits by the theory given by Eqs. (3) and (10).

sponds to the slow relaxation regime where SRPAC is described by the approximation of Eq. (11). At $\sim 240 \mathrm{~K}$ the quantum beats are overdamped so that the anisotropy is equal to zero in the entire experimental time window. Above $240 \mathrm{~K}$ the anisotropy follows an exponential decay with a decay rate decreasing with rising temperature. At the highest measured temperature of $328 \mathrm{~K}$ the decay rate is so small that the anisotropy only very slightly decreases with time. The data above $240 \mathrm{~K}$ correspond to the fast relaxation regime where SRPAC is described by the approximation of Eq. (14). Thus in both limiting regimes, i.e., in the limits of slow and fast relaxation, the measured time evolutions exhibit the characteristic features of SRPAC discussed in connection with Eqs. (11) and (14).

The fit of the SRPAC signal has been performed using the exact theory [Eqs. (3) and (10)] where the perturbation factor of Eq. (10) includes the influence of the rotational relaxation in the frame of the SCM. The parameters of the fit were the relaxation rate $\lambda$, the quadrupole frequency $\Omega$, the effective anisotropy coefficient $A_{22}$, and the intensity at zero time $I_{0}$.

Due to the finite sizes of sample and detector the effective anisotropy coefficient is smaller than the theoretical value of 0.25 . On the other hand, this coefficient depends only on the geometry of the experiment and must be the same for all measurements performed with the same geometrical setup. A value of $A_{22} \simeq 0.19$ was obtained from the fit of the spectra below $200 \mathrm{~K}$ and at $260-280 \mathrm{~K}$. This value was fixed for the other temperatures where $A_{22}$ cannot be obtained unambiguously.

The quadrupole frequency $\Omega$ was extracted from the fit of the spectra in the temperature range from 100 to $220 \mathrm{~K}$ 


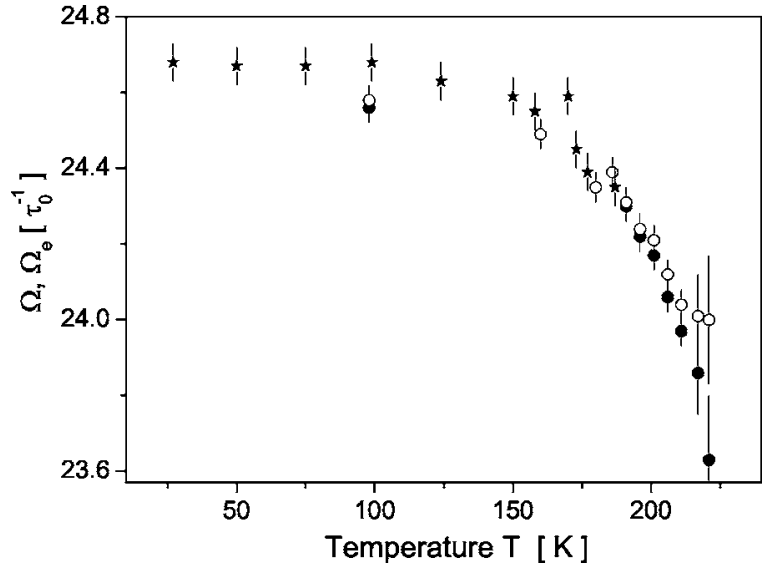

FIG. 9. Temperature dependence of the quadrupole frequency $\Omega$ obtained by SRPAC $(\bigcirc)$ and of the effective quadrupole frequency $\Omega_{e}$ obtained by SRPAC $(\bullet)$ and by NFS $(\star)$.

where the quantum beats are well pronounced. Above this temperature $\Omega$ and $\lambda$ cannot be extracted independently any more. It is seen from Eq. (14) that in the fast relaxation regime only the combination $\Omega^{2} / \lambda$ can be obtained from the experimental data. In Fig. 9 we show the temperature dependence of the quadrupole frequency $\Omega$ and of the effective quadrupole frequency $\Omega_{e}$ which is the frequency of the quantum beats corresponding to the distance between the two quadrupole lines of the Mössbauer spectrum. The difference between $\Omega$ and $\Omega_{e}$ is due to rotational relaxation and is described in the present case by Eq. (12). In addition, the effective quadrupole splitting measured by NFS (Ref. 46) is shown. In principle, no strong variation of the quadrupole frequency is expected in the temperature range studied, since the ${ }^{57} \mathrm{Fe}$ atom is situated well shielded at the center of the FC molecule. Experimentally this tendency is seen up to $100 \mathrm{~K}$ where $\Omega$ is almost constant. Above this temperature the effective quadrupole frequency experiences a noticeable decrease which is seen in both NFS and SRPAC. A similar decrease of the effective quadrupole splitting has been observed in MS studies of the glass transition. ${ }^{44,51,52}$ Such a decrease might be due to a change in molecular geometry, e.g., caused by temperature-dependent solvent-solute interactions. Another explanation of this effect could be the influence of rotational relaxation ${ }^{44}$ which results in an artificial decrease of $\Omega_{e}$ compared with $\Omega$.

The verification of this hypothesis is not possible in the frame of MS or NFS. Here the appearance of translational diffusion leads to an ambiguity in the extraction of the rotational relaxation rate and, therefore, in the extraction of the quadrupole frequency from the spectra. On the other hand, SRPAC allows one to obtain $\Omega$ unambiguously. The comparison of $\Omega$ and $\Omega_{e}$ in Fig. 9 shows that the difference becomes noticeable only above $205 \mathrm{~K}$ and cannot explain the decrease of both $\Omega$ and $\Omega_{e}$ in the range $150-205 \mathrm{~K}$. We rather attribute this decrease to fast librations of the probe molecules in a restricted angular range which leads to a preaverage of the quadrupole splitting. ${ }^{.2}$ In order to extract the relaxation rate $\lambda$ at temperatures above $220 \mathrm{~K}$ we extrapolated $\Omega$ using a linear approximation above $190 \mathrm{~K}$. The real dependence of the quadrupole frequency on temperature

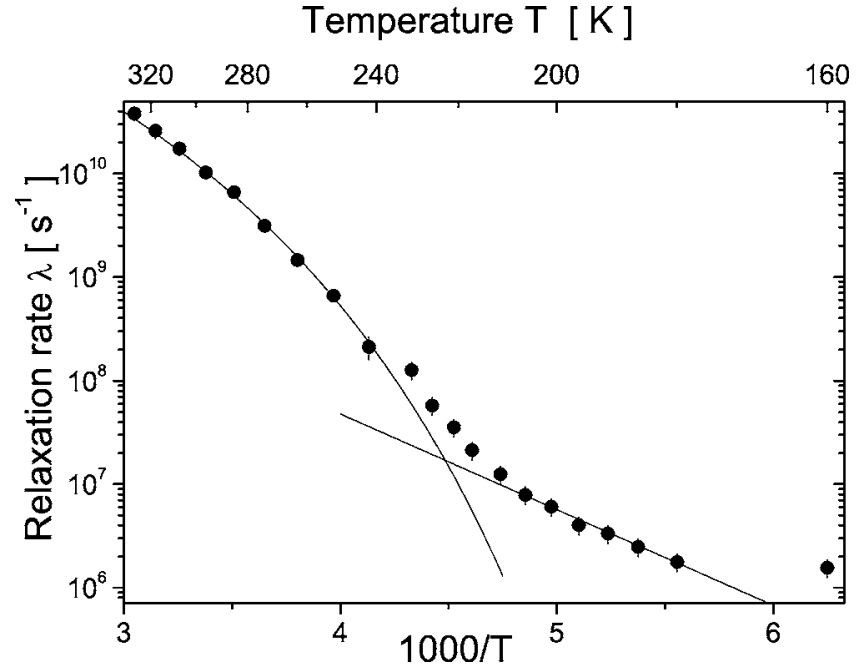

FIG. 10. The relaxation rate $\lambda$ (in $\log$ scale) obtained from SRPAC as a function of inverse temperature 1000/T. The solid lines are the Arrhenius and the Vogel-Fulcher-Tammann fits in the low- and high-temperature regions, respectively. The lower limit of sensitivity is about $1.5 \times 10^{6} \mathrm{~s}^{-1}$ as seen from the data point at $160 \mathrm{~K}$.

can deviate from this extrapolation. However, these possible uncertainties are not significant, because the variation of $\Omega$ with temperature is around a few percent whereas $\lambda$ changes by orders of magnitude.

The relaxation rate $\lambda$ is shown in Fig. 10 as a function of inverse temperature $1000 / T$. It increases from the lower limit of sensitivity of $\sim 1.5 \times 10^{6} \mathrm{~s}^{-1}$ at $180 \mathrm{~K}$ up to $\sim 4 \times 10^{10}$ $\mathrm{s}^{-1}$ at $328 \mathrm{~K}$. As a whole, reliable data are obtained within a frequency range covering more than four decades and within a temperature range covering $150 \mathrm{~K}$ from the glassy state up to the normal liquid state of the glass former.

The relaxation rate exhibits a different behavior above and below $\sim 220 \mathrm{~K}$. At high temperatures it follows a viscosity-like dependence which is typical for dynamics of glass formers and which is widely described by a Vogel-FulcherTammann (VFT) equation ${ }^{53}$

$$
\lambda=\lambda^{(0)} \exp \left(\frac{B}{T_{0}-T}\right) .
$$

We fixed the parameters $B=995 \mathrm{~K}$ and $T_{0}=147 \mathrm{~K}$ according to viscosity measurements in pure DBP $;{ }^{54} \lambda^{(0)}$ was obtained as $8.3 \times 10^{12} \mathrm{~s}^{-1}$. The corresponding curve is shown by the left-hand solid line in Fig. 10. The good coincidence between the temperature dependences of the viscosity of pure DBP and of the relaxation rate of FC molecules dissolved in DBP obtained from SRPAC, as well as the coincidence of the caloric glass-transition temperatures of DBP and DBP-FC, show that the addition of 5\% of FC molecules does not cause noticeable changes in the dynamical properties of DBP.

Below $230 \mathrm{~K}$ the relaxation rate deviates from the VFT dependence and is well fitted in the region below $210 \mathrm{~K}$ by an Arrhenius dependence as shown in Fig. 10 by the righthand solid line, 


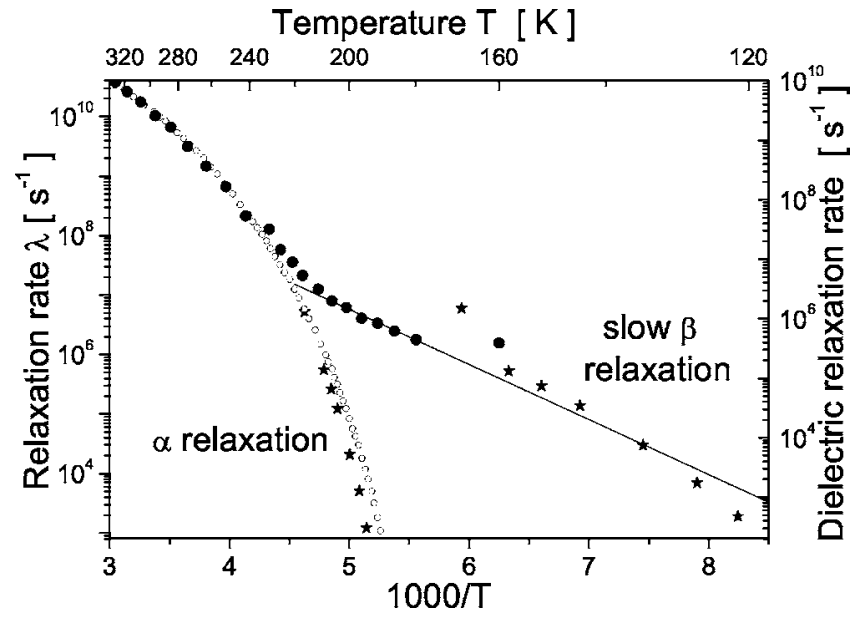

FIG. 11. Inverse temperature dependence of the different relaxation processes (in log scale) in DBP. The left side scale denotes the relaxation rate of the FC molecules obtained from $\operatorname{SRPAC}(\bullet)$. The right side scale denotes the relaxation frequency obtained from dielectric spectroscopy for pure DBP from Ref. $55(\star)$ and Ref. 56 (O). The solid line is the same Arrhenius dependence as in Fig. 10.

$$
\lambda=\lambda^{(0)} \exp \left(-\Delta E / k_{B} T\right)
$$

with $\lambda^{(0)}=2.4 \times 10^{11} \mathrm{~s}^{-1}$ and $\Delta E=17.7 \mathrm{~kJ} \mathrm{~mol}^{-1}$.

In order to clarify the origin of the deviation of the relaxation rate from a VFT dependence we compare in Fig. 11 the relaxation rate obtained from SRPAC with the results of dielectric spectroscopy measurements on pure DBP. ${ }^{55,56}$ In order to neglect the influence of the experimental technique we scale our experimental data and the dielectric frequency to the same value at room temperature, i.e., in the normal liquid regime. At high temperatures the relaxation measured by dielectric spectroscopy consists of a single branch, interpreted as due to $\alpha$ relaxation, which coincides with our data. At low temperatures an additional branch of slower relaxation appears in dielectric spectroscopy, which is usually interpreted as due to slow $\beta$ relaxation. ${ }^{57}$ It follows an Arrhenius temperature dependence and nicely continues the relaxation rates obtained from SRPAC towards lower temperatures. This suggests that the rotational relaxation of the FC probe molecules in the relevant temperature range can be attributed to slow $\beta$ relaxation.

\section{CONCLUSION}

In this paper we apply a new method, synchrotronradiation-based perturbed angular correlations (SRPAC), to investigate hyperfine interactions and dynamics in soft condensed matter. The method can be considered as a bridge between nuclear forward scattering of SR and time differential perturbed angular correlations (TDPAC). SRPAC is independent of the Lamb-Mössbauer factor and allows one to continue investigations of dynamics of Mössbauer isotopes into the liquid state, provided that hyperfine interactions are present. Furthermore, in particular in the case of ${ }^{57} \mathrm{Fe}$, SRPAC yields a much higher anisotropy than TDPAC. ${ }^{11} \mathrm{Be}-$ sides of soft matter, other fields of applications for SRPAC are under investigation, e.g., studies of rotational dynamics in plastic crystals ${ }^{58,59}$ or of hyperfine interactions and dynamics for high energy nuclear transitions ${ }^{60}$ like ${ }^{61} \mathrm{Ni}$.

The theoretical description of SRPAC is similar to that of TDPAC. However, the linear polarization of the synchrotron radiation does not allow us to apply the existing TDPAC approach. Using the general theory of angular correlations we developed an expression that describes the intensity of SRPAC in the case of randomly distributed nuclear spins. The obtained equation is similar to that for TDPAC. In both cases the hyperfine interactions and dynamics are described by the same perturbation factor.

The influence of rotational molecular relaxation on the shape of the SRPAC intensity was analyzed in the frame of the strong collision model which originally was derived for TDPAC. The advantage of this model is that for ${ }^{57} \mathrm{Fe}$ with spin $I=\frac{3}{2}$ an analytical solution can be obtained. This fact allowed us to obtain a rather simple expression for SRPAC in the approximation of slow and fast relaxation and to discuss qualitatively the change of the shape of the SRPAC spectrum due to rotational relaxation.

SRPAC was applied to the molecular glass former dibutyl phthalate doped with $5 \%$ of ferrocene enriched in ${ }^{57} \mathrm{Fe}$. The measurements have been performed in the temperature range from the glassy state up to the normal liquid state of the glass former. To our knowledge, this is the first study of relaxational dynamics of a Mössbauer isotope in the regime of a low-viscosity liquid.

The rotational relaxation rate was obtained in a frequency range from $\sim 1.5 \times 10^{6} \mathrm{~s}^{-1}$ at $180 \mathrm{~K}$ up to $\sim 4 \times 10^{10} \mathrm{~s}^{-1}$ at $328 \mathrm{~K}$, covering more than four decades in magnitude. At high temperatures the relaxation rate has a typical viscouslike temperature behavior whereas below $\sim 210 \mathrm{~K}$ its temperature dependence changes and starts to follow an Arrhenius law. Comparing our results to the data from dielectric spectroscopy for pure DBP, we found that the dynamics of the ferrocene probe molecules reproduces the dynamics of the pure glass former. In particular, the probe dynamics below $210 \mathrm{~K}$ follows the slow $\beta$ relaxation branch of dielectric spectroscopy.

\section{ACKNOWLEDGMENTS}

This work has been supported by the Bundesministerium für Bildung, Wissenschaft, Forschung und Technologie under Contract No. $05 \mathrm{KS} 1 \mathrm{WOC} / 2$. The authors acknowledge stimulating discussions with S. Dattagupta.

\section{APPENDIX: CALCULATION OF THE SRPAC SIGNAL}

Here we derive the time and angular dependence of the SRPAC signal based on the general expression for the angular correlation function developed in Ref. 31 [Eq. (13.137)]

$$
\begin{aligned}
& W\left(\mathbf{k}_{1} \boldsymbol{\sigma}_{1}, \mathbf{k}_{2} \boldsymbol{\sigma}_{2} ; t\right) \\
& \propto \mathrm{e}^{-t / \tau_{0}} \sum_{\lambda, \bar{\lambda}} B_{\lambda q^{\prime}}^{*}(1) A_{\bar{\lambda} \bar{q}^{\prime}}(2) \cdot D_{q^{\prime} q}^{(\lambda)}\left(\mathbf{k}_{1} \boldsymbol{\sigma}_{1} \rightarrow \mathbf{S}\right) \\
& \operatorname{quq}^{\prime} \bar{q}_{\bar{q}, \bar{q}^{\prime}} \\
& \cdot G_{\lambda \bar{\lambda}}^{q \bar{q}}(t) \cdot D_{\bar{q} \bar{q}^{\prime}}^{(\bar{\lambda})}\left(\mathbf{S} \rightarrow \mathbf{k}_{2} \boldsymbol{\sigma}_{2}\right),
\end{aligned}
$$


where $\mathbf{k}_{1(2)}$ and $\boldsymbol{\sigma}_{1(2)}$ are the direction and the polarization of the first (second) $\gamma$ photon; $\lambda, \bar{\lambda}, q, q^{\prime}, \bar{q}, \bar{q}^{\prime}$ are indices of expansion. The angular distribution coefficients $B_{\lambda q^{\prime}}^{*}(1)$ and $A_{\bar{\lambda} \bar{q}^{\prime}}(2)$ are defined by the first and second nuclear transitions and the polarization of the emitted and absorbed radiation. $D_{q^{\prime} q}^{(\lambda)}\left(\mathbf{k}_{1} \boldsymbol{\sigma}_{1} \rightarrow \mathbf{S}\right)$ are the Wigner D functions (rotation matrix elements) which correspond to the rotation of the coordinate system from the one related to the $\gamma$ photon ( $z$ axis along $\mathbf{k}_{1}$, $x$ axis along $\boldsymbol{\sigma}_{1}$ ) to $\mathbf{S}$ usually related to the hyperfine interactions. $D_{\bar{q} \bar{q}^{\prime}}^{(\bar{\lambda})}\left(\mathbf{S} \rightarrow \mathbf{k}_{2} \boldsymbol{\sigma}_{2}\right)$ are the Wigner D functions corresponding to the rotation from $\mathbf{S}$ to $\mathbf{k}_{2} \boldsymbol{\sigma}_{2}$. $G_{\lambda \lambda}^{q \bar{q}}(t)$ is the perturbation factor which describes the time evolution of the intermediate state under the influence of the hyperfine interactions.

For TDPAC (directional correlations) $B_{\lambda q^{\prime}}^{*}(1)$ $=B_{\lambda}(1) \cdot \delta_{q^{\prime} 0}$ and $A_{\bar{\lambda} \bar{q}^{\prime}}(2)=A_{\lambda}^{-}(2) \cdot \delta_{\bar{q}^{\prime} 0}$, which leads to (see Eq. (13.138) in Ref. 31)

$$
\begin{gathered}
W\left(\mathbf{k}_{1}, \mathbf{k}_{2} ; t\right) \propto \mathrm{e}^{-t / \tau_{0}} \sum_{\lambda q, \bar{\lambda} \bar{q}} B_{\lambda}(1) A_{\lambda}^{-}(2) \cdot D_{0 q}^{(\lambda)}\left(\mathbf{k}_{1} \rightarrow \mathbf{S}\right) \\
\cdot G_{\lambda \bar{\lambda}}^{q \bar{q}}(t) \cdot D_{\bar{q} 0}^{(\bar{\lambda})}\left(\mathbf{S} \rightarrow \mathbf{k}_{2}\right)
\end{gathered}
$$

where only the $D_{0 q}^{(\lambda)}$ and $D_{\bar{q} 0}^{(\bar{\lambda})}$ matrix elements exist which can be expressed via the spherical harmonics $Y_{\lambda q}$ and $Y_{\bar{\lambda} \bar{q}}^{-} A_{\lambda}^{-}(2)$ and $B_{\lambda}(1)$ depend only on the nuclear transition and can be easily calculated according to Eqs. (12.228), (12.182), and (12.168) in Ref. 31.

For SRPAC $A_{\bar{\lambda} \bar{q}^{\prime}}(2)$ is expressed in a similar way. $B_{\lambda q^{\prime}}^{*}(1)$, however, is more complicated due to the linear polarization of the synchrotron radiation:

$$
\begin{aligned}
& B_{\lambda 0}^{*}(1)=B_{\lambda}(1), \quad B_{\lambda \pm 1}^{*}(1)=0, \\
& B_{\lambda \pm 2}^{*}(1)=\frac{1}{4} \sqrt{\frac{(\lambda+2) !}{(\lambda-2) !}} B_{\lambda}(1) .
\end{aligned}
$$

In order to represent the SRPAC signal in a form similar to Eq. (A2) we proceed as follows: We separate the rotation $\mathbf{k}_{1} \boldsymbol{\sigma}_{1} \rightarrow \mathbf{S}$ in two rotations $\mathbf{k}_{1} \boldsymbol{\sigma}_{1} \rightarrow \mathbf{S}_{0} \rightarrow \mathbf{S}$ where we choose a coordinate system $\mathbf{S}_{0}$ with $z$ axis perpendicular to the plane of the synchrotron ring (directed along the magnetic polarization of $\gamma_{1}$ ). Then the rotation $\mathbf{k}_{1} \boldsymbol{\sigma}_{1} \rightarrow \mathbf{S}_{0}$ is the rotation around the $z$ axis by $\pi / 2$ plus the rotation around the new $y$ axis by $\pi / 2$ plus the rotation by $\eta$ around the new $z$ axis. We can replace the first Wigner D function in Eq. (A1) by

$$
D_{q^{\prime} q}^{(\lambda)}\left(\mathbf{k}_{1} \boldsymbol{\sigma}_{1} \rightarrow \mathbf{S}\right)=\sum_{m} D_{q^{\prime} m}^{(\lambda)}\left(\frac{\pi}{2}, \frac{\pi}{2}, \eta\right) \cdot D_{m q}^{(\lambda)}\left(\mathbf{S}_{0} \rightarrow \mathbf{S}\right)
$$

and replace the sum over $q^{\prime}$ in Eq. (A1) by

$$
\begin{aligned}
\sum_{q^{\prime}} B_{\lambda q^{\prime}}^{*}(1) \cdot D_{q^{\prime} q}^{(\lambda)}\left(\mathbf{k}_{1} \boldsymbol{\sigma}_{1} \rightarrow \mathbf{S}\right) \\
\quad=\sum_{m}\left[\sum_{q^{\prime}} B_{\lambda q^{\prime}}^{*}(1) D_{q^{\prime} m}^{(\lambda)}\left(\frac{\pi}{2}, \frac{\pi}{2}, \eta\right)\right] \cdot D_{m q}^{(\lambda)}\left(\mathbf{S}_{0} \rightarrow \mathbf{S}\right) .
\end{aligned}
$$

The expression in brackets can be replaced using Eq. (A3) and the presentation of the Wigner $\mathrm{D}$ function:

$$
D_{q^{\prime} m}^{(\lambda)}\left(\frac{\pi}{2}, \frac{\pi}{2}, \eta\right)=\mathrm{e}^{i q^{\prime} \pi / 2} \cdot d_{q^{\prime} m}^{(\lambda)}(\pi / 2) \cdot \mathrm{e}^{i m \eta} .
$$

Therefore

$$
\begin{aligned}
\sum_{q^{\prime}=0, \pm 2} & B_{\lambda q^{\prime}}^{*}(1) D_{q^{\prime} m}^{(\lambda)}\left(\frac{\pi}{2}, \frac{\pi}{2}, \eta\right) \\
= & B_{\lambda}(1) \cdot \mathrm{e}^{l m \eta} \cdot\left\{d_{0 m}^{(\lambda)}\left(\frac{\pi}{2}\right)-\frac{1}{4} \sqrt{\frac{(\lambda+2) !}{(\lambda-2) !}}\right. \\
& \left.\times\left[d_{-2 m}^{(\lambda)}\left(\frac{\pi}{2}\right)+d_{2 m}^{(\lambda)}\left(\frac{\pi}{2}\right)\right]\right\} .
\end{aligned}
$$

The last expression can be calculated using, e.g., tables ${ }^{32}$ for $d_{n m}^{(\lambda)}(\pi / 2)$. The value of $\lambda$ can be only even because of the polarization properties of the radiation. We obtain that Eq. (A7) is equal to $B_{\lambda}(1) p_{\lambda} \delta_{m 0}$ with $p_{\lambda}=1,-2$ for $\lambda=0,2$. The proportionality of this expression to $\delta_{m 0}$ simplifies the further calculations. However, this is valid only for $\lambda=0,2$. Therefore we limit the consideration here to the case of a multipolarity of the nuclear transition $L=1$, since $\lambda, \bar{\lambda}$ $\leqslant \min \left(2 L, 2 I_{e}\right)$. As a result, Eq. (A5) can be replaced by

$$
\sum_{q^{\prime}} B_{\lambda q^{\prime}}^{*}(1) \cdot D_{q^{\prime} q}^{(\lambda)}\left(\mathbf{k}_{1} \boldsymbol{\sigma}_{1} \rightarrow \mathbf{S}\right)=p_{\lambda} B_{\lambda}(1) D_{0 q}^{(\lambda)}\left(\mathbf{S}_{0} \rightarrow \mathbf{S}\right) .
$$

Inserting this result into Eq. (A1) and, similar to the procedure in TDPAC, replacing $A_{\bar{\lambda} \bar{q}^{\prime}}^{-}(2)$ by $A_{\lambda}^{-}(2) \cdot \delta_{\bar{q}^{\prime} 0}$ we obtain the angular correlation function for SRPAC:

$$
\begin{gathered}
W\left(\mathbf{k}_{2} ; t\right) \propto \mathrm{e}^{-t / \tau_{0}} \sum_{\substack{\lambda \bar{\lambda}=0,2 \\
q \bar{q}}} p_{\lambda} B_{\lambda}(1) A_{\lambda}^{-}(2) \cdot D_{0 q}^{(\lambda)}\left(\mathbf{S}_{0} \rightarrow \mathbf{S}\right) \\
\cdot G_{\lambda \bar{\lambda}}^{q \bar{q}}(t) \cdot D_{\bar{q} 0}^{(\bar{\lambda})}\left(\mathbf{S} \rightarrow \mathbf{k}_{2}\right) .
\end{gathered}
$$

This expression is similar to Eq. (A2) describing the directional correlations in TDPAC. The difference is the restriction of the sum to $\lambda, \bar{\lambda} \leqslant 2$, the additional coefficient $p_{\lambda}=1$, -2 for $\lambda=0,2$, and the replacement of $\mathbf{k}_{1}$ by $\mathbf{S}_{0}$, i.e., the replacement of the direction of the incoming SR $\gamma$-photon by the direction of its magnetic polarization. Taking these differences into account one can apply the theory available for TDPAC to SRPAC.

In the case of a single multipolarity of the nuclear transition one can replace $B_{\lambda}(1)$ by $A_{\lambda}(1)$. The first and the second nuclear transition are in SRPAC the same transitions so that $A_{\lambda}(1)=A_{\lambda}(2) \equiv A_{\lambda}$, which allows one to introduce the aniso- 
tropy coefficient $A_{\lambda \lambda} \bar{\lambda} \equiv B_{\lambda}(1) A_{\lambda}^{-}(2)=A_{\lambda} A_{\lambda}^{-}$. For isotropic hyperfine interactions of an ensemble of nuclei the perturbation factor transforms as

$$
G_{\lambda \lambda}^{q \bar{q}}(t) \rightarrow \frac{\delta_{\lambda \lambda}^{-} \delta_{q \bar{q}}}{2 \lambda+1} \sum_{m} G_{\lambda \lambda}^{m m}(t) \equiv \delta_{\lambda \lambda} \delta_{q \bar{q}} \cdot G_{\lambda \lambda}(t)
$$

Therefore for isotropic hyperfine interactions the SRPAC signal $I(\vartheta ; t)$ is given as

$$
I(\vartheta ; t)=I_{0} \cdot W\left(\mathbf{k}_{2} ; t\right)=I_{0} \mathrm{e}^{-t / \tau_{0}} \cdot\left[1-2 A_{22} G_{22}(t) P_{2}(\cos \vartheta)\right]
$$

where $I_{0}$ is proportional to the incoming intensity; $\vartheta$ is the angle between the vertical axis (direction of the magnetic polarization of the incoming photon) and the direction of the scattered photon; and $P_{2}(x)$ is the Legendre polynomial of second order. Here we use the property of the Wigner functions that

$$
\sum_{q} D_{0 q}^{(\lambda)}\left(\mathbf{S}_{0} \rightarrow \mathbf{S}\right) \cdot D_{q 0}^{(\lambda)}\left(\mathbf{S} \rightarrow \mathbf{k}_{2}\right)=D_{00}^{(\lambda)}\left(\mathbf{S}_{0} \rightarrow \mathbf{k}_{2}\right)=P_{2}(\cos \vartheta) .
$$

*Electronic address: sergueev@esrf.fr

${ }^{1}$ For an introduction see, e.g., G. K. Wertheim, Mössbauer Effect: Principles and Applications (Academic Press, New York, 1964).

${ }^{2}$ For recent applications see, e.g., the Proceedings of the last ICAME conferences in Hyperfine Interact. 156/157 (2004), 141/ 142 (2002), and 126 (2000).

${ }^{3}$ P. A. Flinn, in Applications of Mössbauer Spectroscopy, Vol. II, edited by R. L. Cohen (Academic Press, New York, 1980), p. 393.

${ }^{4}$ E. R. Bauminger and I. Nowik, in Mössbauer Spectroscopy, edited by D. P. E. Dickson and F. J. Berry (Cambridge University Press, Cambridge, 1986), p. 219.

${ }^{5}$ G. U. Nienhaus and F. Parak, Hyperfine Interact. 90, 243 (1994).

${ }^{6}$ A. Gahl, M. Hillberg, F. J. Litterst, T. Pohlmann, C. Hubsch, O. Nuyken, F. Garwe, M. Beiner, and E. Donth, J. Phys.: Condens. Matter 10, 961 (1998).

${ }^{7}$ R. H. Herber and I. Nowik, Hyperfine Interact. 126, 127 (2000).

${ }^{8}$ V. Ya. Rochev, in Advances in Liquid Crystals Vol. 5, edited by G. H. Brown (Academic Press, New York, 1982).

${ }^{9}$ For an introduction see, e.g., T. Butz, Z. Naturforsch., A: Phys. Sci. 51, 396 (1996).

${ }^{10}$ For recent applications see, e.g., the Proceedings of the last HI conferences in Hyperfine Interact. in press (2005), 136/137 (2001), and 120/121 (1999), and of the last NQI symposia in Hyperfine Interact. in press (2005), Z. Naturforsch., A: Phys. Sci57 (2002) and 55 (2000).

${ }^{11}$ For instance, in TDPAC with ${ }^{57} \mathrm{Fe}$ the $122-14 \mathrm{keV}$ cascade gives an anisotropy of only $2 \%$ [see C. Hohenemser, R. Reno, H. C. Benski, and J. Lehr, Phys. Rev. 184, 298 (1969)], whereas SRPAC yields an anisotropy of $\sim 40 \%$ [see Eq. (5) and Fig. 7].

${ }^{12}$ I. Sergueev, U. van Bürck, A. I. Chumakov, T. Asthalter, G. V. Smirnov, H. Franz, R. Rüffer, and W. Petry, Ann. Report TUM Physik-Dep. E13, 39 (2001), URL http://www.e13.physik.tumuenchen.de/jahresbericht/jabe2001.pdf (unpublished).

${ }^{13}$ I. Sergueev, U. van Bürck, A. I. Chumakov, T. Asthalter, G. V. Smirnov, H. Franz, R. Rüffer, and W. Petry, Highlights ESRF 2002, 61 (2003), URL http://www.esrf.fr/UsersAndScience/ Publications/Highlights/2002/HRRS/HRRS11/ (unpublished).

${ }^{14}$ G. T. Trammell and J. P. Hannon, Phys. Rev. B 18, 165 (1978); Phys. Rev. B 19, 3835(E) (1979).

${ }^{15}$ A. Q. R. Baron, A. I. Chumakov, S. L. Ruby, J. Arthur, G. S. Brown, G. V. Smirnov, and U. van Bürck, Phys. Rev. B 51, 16
384 (1995)

${ }^{16}$ E. M. Iolin, Hyperfine Interact. 1, 513 (1996).

${ }^{17}$ E. Matthias, M. G. White, E. D. Poliakoff, R. A. Rosenberg, S. T. Lee, and D. A. Shirley, Chem. Phys. Lett. 54, 30 (1978).

${ }^{18}$ Nuclear Resonant Scattering of Synchrotron Radiation, edited by E. Gerdau and H. de Waard; Hyperfine Interact. 123/124 (1999), 125 (2000).

${ }^{19}$ E. Gerdau, R. Rüffer, H. Winkler, W. Tolksdorf, C. P. Klages, and J. P. Hannon, Phys. Rev. Lett. 54, 835 (1985).

${ }^{20}$ J. B. Hastings, D. P. Siddons, U. van Bürck, R. Hollatz, and U. Bergmann, Phys. Rev. Lett. 66, 770 (1991).

${ }^{21}$ U. Bergmann, J. B. Hastings, and D. P. Siddons, Phys. Rev. B 49, R1513 (1994).

${ }^{22}$ M. Seto, Y. Yoda, S. Kikuta, X. W. Zhang, and M. Ando, Phys. Rev. Lett. 74, 3828 (1995).

${ }^{23}$ W. Sturhahn, T. S. Toellner, E. E. Alp, X. Zhang, M. Ando, Y. Yoda, S. Kikuta, M. Seto, C. W. Kimball, and B. Dabrowski, Phys. Rev. Lett. 74, 3832 (1995).

${ }^{24}$ A. I. Chumakov, R. Rüffer, H. Grünsteudel, H. F. Grünsteudel, G. Grübel, J. Metge, O. Leupold, and H. A. Goodwin, Europhys. Lett. 30, 427 (1995).

${ }^{25}$ A. Q. R. Baron, A. I. Chumakov, R. Rüffer, H. F. Grünsteudel, and O. Leupold, Europhys. Lett. 34, 331 (1996).

${ }^{26}$ M. Seto, S. Kitao, Y. Kobayashi, R. Haruki, Y. Yoda, T. Mitsui, and T. Ishikawa, Phys. Rev. Lett. 91, 185505 (2003).

${ }^{27}$ S. Dattagupta, Radiat. Phys. Chem. 70, 511 (2004).

${ }^{28}$ Yu. Kagan, A. M. Afanas'ev, and V. G. Kohn, J. Phys. C 12, 615 (1979).

${ }^{29}$ R. M. Steffen and H. Frauenfelder, in Perturtbed Angular Correlations, edited by E. Karlsson, E. Matthias, and K. Siegbahn (North-Holland, Amsterdam, 1964), p. 1.

${ }^{30} \mathrm{H}$. Frauenfelder and R. M. Steffen, in Alpha-, Beta- and GammaRay Spectroscopy, Vol. 2, edited by K. Siegbahn (NorthHolland, Amsterdam, 1965), p. 997.

${ }^{31}$ R. M. Steffen and K. Alder, in The Electromagnetic Interaction in Nuclear Spectroscopy, edited by W. D. Hamilton (NorthHolland, Amsterdam, 1975), pp. 505, 583.

${ }^{32}$ D. A. Varshalovich, A. N. Moskalev, and V. K. Khersonskii, Quantum Theory of Angular Momentum (World Scientific, Singapore, 1988).

${ }^{33}$ A. Abragam and R. V. Pound, Phys. Rev. 92, 943 (1953).

${ }^{34}$ M. Blume, Phys. Rev. 174, 351 (1968). 
${ }^{35}$ R. Kubo, J. Phys. Soc. Jpn. 9, 935 (1954).

${ }^{36}$ S. Dattagupta, Hyperfine Interact. 11, 77 (1981).

${ }^{37}$ R. M. Lynden-Bell, Mol. Phys. 21, 891 (1971).

${ }^{38}$ H. Winkler, Z. Phys. A 276, 225 (1976).

${ }^{39}$ G. Diezemann and H. Sillescu, J. Chem. Phys. 111, 1126 (1999).

${ }^{40} \mathrm{~S}$. Dattagupta, Relaxation Phenomena in Condensed Matter Physics (Academic Press, Orlando, 1987).

${ }^{41}$ R. M. Lynden-Bell, Mol. Phys. 26, 979 (1973).

${ }^{42}$ I. Sergueev, Ph.D. thesis, TU München, 2004, URL http:// tumb1.biblio.tu-muenchen.de/publ/diss/ph/2004/sergueev.html.

${ }^{43}$ A. G. Marshall and C. F. Meares, J. Chem. Phys. 56, 1226 (1972).

${ }^{44}$ S. L. Ruby, B. J. Zabransky, and P. A. Flinn, J. Phys. (France) 37, C6-745 (1976).

${ }^{45}$ A. Meyer, H. Franz, J. Wuttke, W. Petry, N. Wiele, R. Rüffer, and C. Hübsch, Z. Phys. B: Condens. Matter 103, 479 (1997).

${ }^{46}$ I. Sergueev, H. Franz, T. Asthalter, W. Petry, U. van Bürck, and G. V. Smirnov, Phys. Rev. B 66, 184210 (2002).

${ }^{47}$ A. I. Chumakov, I. Sergueev, U. van Bürck, W. Schirmacher, T. Asthalter, R. Rüffer, O. Leupold, and W. Petry, Phys. Rev. Lett. 92, 245508 (2004).

${ }^{48}$ R. Rüffer and A. I. Chumakov, Hyperfine Interact. 97/98, 589 (1996).
${ }^{49}$ H. Franz, B. Hukelmann, and J. R. Schneider, Hyperfine Interact. 126, 397 (2000).

${ }^{50}$ A. Q. R. Baron, Nucl. Instrum. Methods Phys. Res. A 352, 665 (1995).

${ }^{51}$ A. Abras and J. G. Mullen, Phys. Rev. A 6, 2343 (1972).

${ }^{52}$ R. Brüning, D. H. Ryan, and J. O. Ström-Olsen, Hyperfine Interact. 55, 911 (1990).

${ }^{53}$ See, e.g., C. A. Angell, K. L. Ngai, G. B. McKenna, P. F. McMillan, and S. W. Martin, J. Appl. Phys. 88, 3113 (2000).

${ }^{54}$ N. Menon, S. R. Nagel, and D. C. Venerus, Phys. Rev. Lett. 73, 963 (1994).

${ }^{55}$ S. A. Dzyuba and Yu. D. Tsvetkov, J. Struct. Chem. 28, 343 (1987).

${ }^{56}$ P. K. Dixon, L. Wu, S. R. Nagel, B. D. Williams, and J. P. Carini, Phys. Rev. Lett. 65, 1108 (1990).

${ }^{57}$ A. Kudlik, S. Benkhof, T. Blochowicz, C. Tschirwitz, and E. Rössler, J. Mol. Struct. 479, 201 (1999).

${ }^{58}$ T. Asthalter, I. Sergueev, and U. van Bürck, J. Phys. Chem. Solids 66, 2271 (2005).

${ }^{59}$ T. Asthalter, I. Sergueev, U. van Bürck, and R. Dinnebier (unpublished).

${ }^{60}$ O. Leupold (unpublished). 